\title{
FUZZY BASED CONTROL USING LabVIEW FOR MISO TEMPERATURE PROCESS
}

\author{
B.Chinthamani ${ }^{1}$, P. T. Rajeena Mol $^{2}$, K.P.Kamini ${ }^{3}$, K.R.Sughashini ${ }^{4}$ \\ 1, 2,3,4 Assistant Professor, Dept of Electronics and Instrumentation Engineering, Easwari Engineering College \\ Chinthu_vishal@yahoo.co.in, rajeenamol2009@gmail.com,Kaminipalani@yahoo.com,sughashinikrme@gmail.com
}

\begin{abstract}
This project aims at designing and implementing a fuzzy controller for Multiple Input Single Output temperature process. Temperature control of water in the tank is achieved by varying current to the heating rod and inlet flow rate by a fuzzy controller. The system consists of a tank, reservoir, variable speed pump, temperature sensor placed inside a heating tank containing the heating rod, voltage controlled current source and computer. Water is pumped into the tank from reservoir and RTD measures the current temperature. The signal from the temperature sensor is sent to the DAQ interfaced to the computer. LabVIEW software is used to acquire the input signal and send the output signal that is determined by the control algorithm. Fuzzy logic controller is designed in LabVIEW. Based on the set point temperature, the controller sets the appropriate current to the heating rod. If the required temperature is less than that sensed by the temperature sensor, the flow rate of water into the tank is controlled by a variable speed pump. While conventional controllers are analytically described by a set of equations, the FLC is described by a knowledge-based algorithm. Thus this system is highly efficient in both heating and reducing the temperature of the tank. A fuzzy logic controller gives faster response, is more reliable and recovers quickly from system upsets. It also works well to uncertainties in the process variables and it does not require mathematical modelling.
\end{abstract}

\section{INTRODUCTION}

\section{Temperature Control}

Process control system is made up of a group of electronic devices and equipments that provides stability, accuracy and eliminates harmful transition statuses in production process. Process control is extensively used in industry and enables mass production of continuous processes.

Temperature control of water is a process in which change of temperature is measured and the passage of heat energy into or out of it is adjusted to achieve a desired temperature. It is the basic requirement in domestic as well as industrial applications as it provides a critical condition for combustion, chemical reaction, fermentation, drying, calcinations, distillation etc. Poor temperature control can cause major safety, quality and productivity problems.

At the beginning of the industrial revolution, especially during 60 's and 70's relays were used to operate automatic machines and they were interconnected using wires inside control panel, hence the control became complex. Programmable logic controller is an industrial computer used for automation of processes. To discover an error in the system much time was required especially with more complex process control system. These panels proved to be inflexible and needs for reliable and rigid controller enhanced rapidly thereby resulting in the invention of new technologies and softwares.

\section{CONTROLLER}

To accurately control process temperature without extensive operator involvement, a temperature control system relies upon a controller, which accepts input from temperature sensors such as a thermocouple or RTD. It compares the actual temperature to the desired control temperature, or set point, and provides an output to a control element.

The following items should be considered when selecting a controller:

- Type of input sensor (thermocouple, RTD) and temperature range

- Type of output required (electromechanical relay, SSR, analog output)

- Control algorithm needed (on/off, proportional, PID)

- Number and type of outputs (heat, cool, alarm, limit)

Once the model for a process has been developed, then there is a need for control action to maintain the process under steady state.

\section{DRAWBACKS OF THE EXISTING SYSTEM}

The existing system needs the employment of instrumentation engineers to monitor and control the various processes. The existing system does not show the real time simulation; thereby having the text based programming complexity and also having difficulty in identifying the errors and rectifying it. The use of the LabVIEW software helps in overcoming these problems 
and makes the processes done in a simpler and easier manner. In a general temperature control process, cooling process takes time i.e., the heater power is decreased or is turned off in order to achieve decrease in water temperature.

\section{NEED FOR THE PROJECT}

The need for today's industry is a real time monitoring and control of the various parameters in a simpler manner with easy identification and rectification of errors. As the existing system's cooling process is time consuming, a need to speed up the cooling process arises.

\section{PROPOSED SYSTEM}

In this project, temperature monitoring and control of water in the tank is established with the help of fuzzy controller designed in LabVIEW software. This implementation attempts to correct the error between the measured temperature and the desired set point thus achieving efficient temperature control.

\section{METHODOLOGY}

Temperature control of water is a process in which change of temperature of water is measured or otherwise detected, and the passage of heat energy into or out of the water is adjusted to achieve the desired temperature. The fuzzy controller uses defined rules to control a fuzzy system based on the current values of input variables, temperature error and error rate. Triangular form of membership function is used here. It involves the use of LabVIEW software which has enhanced features employed in this method for continuously monitoring the process even when the process variable reaches the set point. The parameters adjust themselves to the conditions of the process automatically as the process variable changes and thereby the output is attained.

\section{Fuzzy Logic}

The MISO temperature control system is designed and implemented using fuzzy controller that is programmed in LabVIEW. Fuzzy logic is a method of rule-based decision making used for process control.

\section{Implementation Of Fuzzy Logic}

Fuzzy system consists of three main parts: linguistic variables, membership functions and rules. The basic steps in designing fuzzy logic control is as follows:

- Identifying the input and output variables.

- Partitioning the interval of each input and output into number of fuzzy subsets, assigning each a linguistic label.

- Determining a membership function for each fuzzy subset.

- Assigning the fuzzy relationship between the "input fuzzy subsets" on one hand and the "output fuzzy subsets" on the other hand, thus forming the RuleBase.

- Interpreting the rules using fuzzy "AND" and "OR", operators. In fuzzy systems, more than one rule may fire at the same time, but with varied strengths.

- Translating the processed fuzzy data into the crisp data suitable for real time applications.

\section{CLOSED LOOP TEMPERATURE CONTROL}

The system parameter which is the process variable that needs to be controlled is temperature $\left({ }^{\circ} \mathrm{C}\right)$. Continuous outflow of water from the tank acts as a disturbance to the process. A sensor is used to measure the process variable and provide feedback to the control system. The set point is the desired or reference value for the process variable. At any given moment, the difference between the process variable and set point is used by the control system algorithm ( compensator ), to determine the desired actuator output to drive the system (plant). For instance, if the measured temperature process variable is $40{ }^{\circ} \mathrm{C}$ and desired temperature set point is $50{ }^{\circ} \mathrm{C}$, then the actuator output specified by the control algorithm is to drive a heater.

Actuator signal to drive a heater causes the system to become warmer which results in an increase in the temperature process variable whereas the actuator signal to drive the pump causes the system to reduce temperature more efficiently. This is called a closed loop control system, because the process of reading sensors to provide constant feedback and calculating the desired actuator output is repeated continuously and at a fixed loop rate.

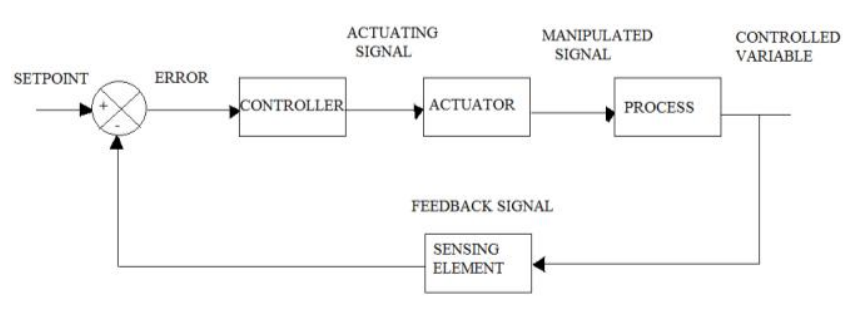

Fig1: closed loop control

\section{Control Configuration}

A control configuration is the information structure that is used to connect the available measurements to the available manipulated variables. Depending on the number of controlled output and manipulated input we have in a chemical process, we can distinguish the control configurations as :

(i)Single input single output(SISO)

(ii)Multiple input multiple output(MIMO)

(iii)Multiple input single output(MISO)

(iv)Single input multiple output(SIMO) control systems. 


\section{MULTIPLE INPUT SINGLE OUTPUT PROCESS}

If there is only one control variable and more than one manipulated variable the control configuration is called multiple input single output (MISO) system. For example, the temperature of the reactor feed (a disturbance) can be measured, and the coolant flow rate can be manipulated. Both of these affect the reactor temperature.

\section{Process Setup}

The process setup consists of a single tank filled with water and a heater is being incorporated in order to supply heat. Initially inflow and outflow of water is set at a constant flow rate. A Resistance Temperature Detector (RTD) is used to measure the temperature of the setup. This signal is conditioned and then fed to the analog input module of the DAQ, which in turn sends the signal to LabVIEW. The RTD gives the output in ohms and by signal conditioning it, output is received in volts $(0-5 \mathrm{~V})$. The LabVIEW compares this signal with the set point and generates a digital signal which is fed to the analog output module of the DAQ. The output from this module is given to the heater control unit and the pump through SCR and SSR .The purpose of the proportional SSR and SCR is to vary the current through the heater and the pump respectively.

The schematic diagram of the process setup is shown in Fig.2

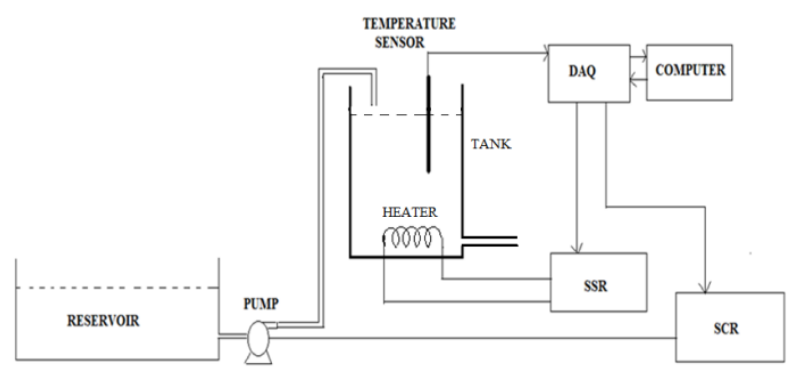

Fig2:schematic diagram of process setup

\section{TEMPERATURE SENSOR}

Resistance temperature detectors are used to measure temperature by correlating the resistance of the RTD element with temperature. The RTD element is made from a pure material whose resistance at various temperatures has been documented. The material has a predictable change in resistance as the temperature changes and it is this predictable change that is used to determine temperature. The material used here is Platinum.

A platinum resistance temperature detector (RTD) Pt100 is a device with a typical resistance of $100 \Omega$ at $0^{\circ} \mathrm{C}$ (it is called Pt100). It changes resistance value as its temperature changes following a positive slope (resistance increases when temperature is increasing).
While thermocouples use the Seebeck effect to generate a voltage, resistance thermometers use electrical resistance and require a power source to operate. The resistance ideally varies linearly with temperature.

For a PT100 sensor, a $1{ }^{\circ} \mathrm{C}$ temperature change will cause a $0.384 \mathrm{ohm}$ change in resistance. RTD output for the corresponding temperature has been tabulated in Table 4.2

Table1: RTD output for corresponding temperature

\begin{tabular}{|c|c|}
\hline TEMPERATURE & RTD OUTPUT $(\Omega)$ \\
\hline 0 & 100 \\
\hline 20 & 107.79 \\
\hline 40 & 115.54 \\
\hline 50 & 119.40 \\
\hline 60 & 123.24 \\
\hline
\end{tabular}

So even a small error in measurement of the resistance (for example, the resistance of the wires leading to the sensor) can cause a large error in the measurement of the temperature. For precision work, sensors have four wires- two to carry the sense current, and two to measure the voltage across the sensor element. The linear characteristics of RTD is shown in the Figure3

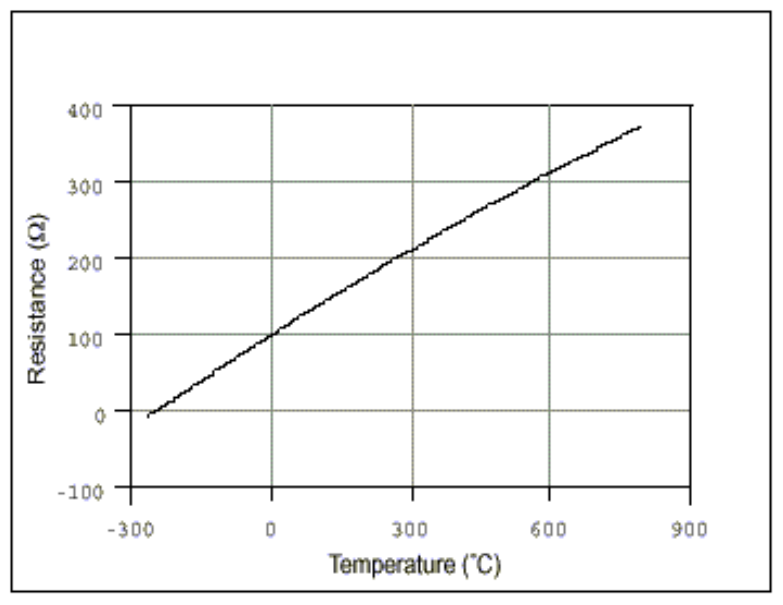

Fig3: characteristics of RTD

\section{RTD SIGNAL CONDITIONING CIRCUIT}

Signal conditioning means manipulating an analog signal such that it measures the requirements of next stage for further processing. For example, the output of an electronic temperature sensor, which is probably too low for an Analogto-Digital converter (ADC) to process directly. In this case the signal conditioning amplification is necessary to bring the voltage level up to that required by a ADC. More generally, the signal conditioning can include amplification, ,converting and any other process required to make sensor output suitable for conversion to digital format. 
The circuit diagram of RTD conditioning is shown in figure4

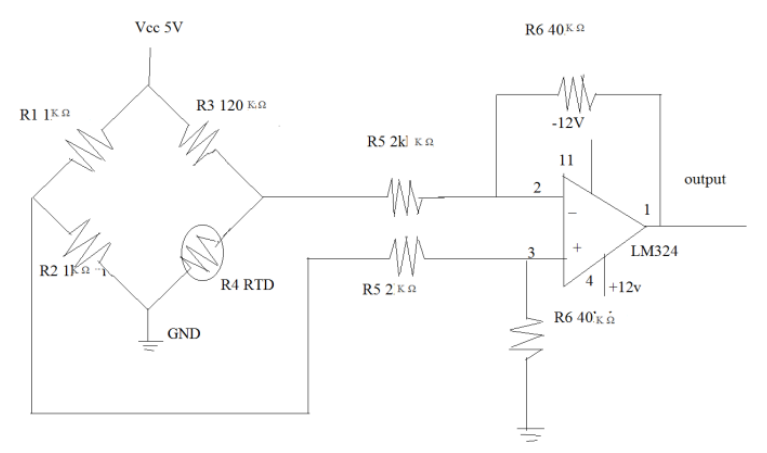

Fig4: signal conditioning of RTD

\section{CIRCUIT DESIGN}

$\mathrm{V} 1=(\mathrm{R} 1 / \mathrm{R} 1+\mathrm{R} 2) * \mathrm{Vcc}$

$\mathrm{V} 2=(\mathrm{R} 3 / \mathrm{R} 3+\mathrm{R} 4) * \mathrm{Vcc}$

V1-V2=Bridge Output Voltage

Gain=R6/R5

\section{Calculation:}

$\mathrm{V} 1=(1 \mathrm{k} \Omega / 1 \mathrm{k} \Omega+1 \mathrm{k} \Omega) * 5 \mathrm{~V}=2.5 \mathrm{~V}$

$\mathrm{V} 2=(120 \Omega / 120 \Omega+121 \Omega) * 5 \mathrm{~V}=2.48 \mathrm{~V}$

$\mathrm{V} 1-\mathrm{V} 2=0.02 \mathrm{~V}$

Gain for differential amplifier

$\mathrm{R} 6 / \mathrm{R} 5=40 \mathrm{k} \Omega / 2 \mathrm{k} \Omega=20$

Output Voltage $=$ Gain $*$ Bridge Output $=20 * 0.02=0.4 \mathrm{~V}$

Bridge output varies from 0.02 to $0.2 \mathrm{~V}$

At $38 \mathrm{C}$, the output is $0.4 \mathrm{~V}$

At $100 \mathrm{C}$, the output is $4 \mathrm{~V}$

\section{FUZZY SYSTEM}

A fuzzy system is a system of variables that are associated using fuzzy logic. A fuzzy controller uses defined rules to control a fuzzy system based on the current values of input variables. Fuzzy system consists of three main parts: linguistic variables, membership functions and rules.

\section{Linguistic Variables And Terms}

Linguistic variables represent, in words, the input variables and output variables of the system to be controlled. Linguistic variables usually have an odd number of linguistic terms, with a middle linguistic term and symmetric linguistic terms at each extreme. Each linguistic variable has a range of expected values. The linguistic variables current temperature and desired temperature each might include the linguistic terms cold, moderate, and hot. The linguistic variable heater setting might include the linguistic terms off, low, and high.

\section{Membership Functions}

Membership functions are numerical functions corresponding to linguisticterms. A membership function represents the degree of membership of linguistic variables within their linguistic terms. The degree of membership is continuous between 0 and 1 , where 0 is equal to $0 \%$ membership and 1 is equal to $100 \%$ membership. There are several types of membership functions available, namely, $\Lambda$-type (triangular shape), П-type (trapezoidal shape), singleton-type (vertical line shape), Sigmoid-type (wave shape), and Gaussian-type (bell shape) membership functions. The various membership functions are shown in Fig5.

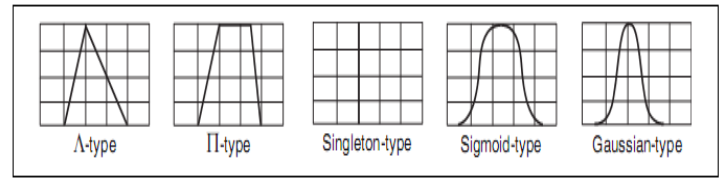

Fig5: various membership functions

\section{Fuzzy Inputs and Output}

In our project there are two inputs and based on the combination of these inputs, the output is obtained. Each of these inputs has seven subsets.

Input 1: Temperature Error

Input 2: Temperature Error Rate

Output : Fuzzy output to Heating rod or Pump

\section{Subsets For Inputs And Output}

Input 1(Temperature Error) : Positive Big, Positive Medium, Positive Small, Zero, Negative Small, Negative Medium, Negative Big

Figure 6 displays the Edit Variable dialog box

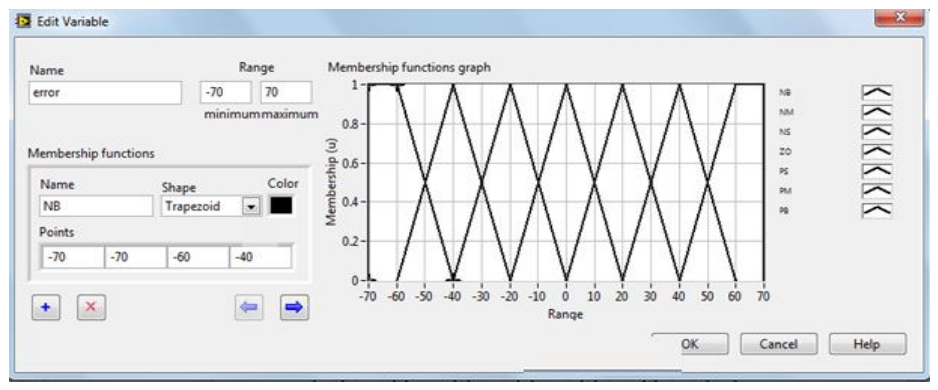

Fig6: membership function for error 
Input 2(Temperature Error Rate) : Positive Big, Positive Medium, Positive Small, Zero, Negative Small, Negative Medium, Negative Big

Figure 7 displays the Edit Variable dialog box with all members hifunctions for the Error rate input variable.

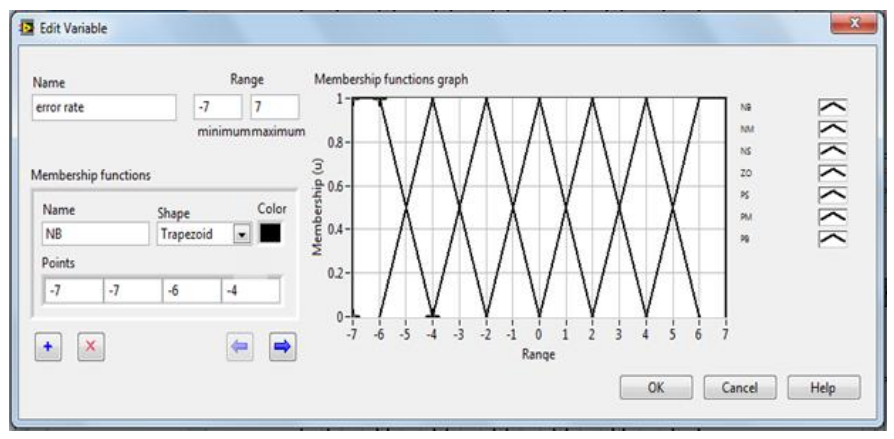

Fig7: membership function for error rate

Output : Positive Big, Positive Medium, Positive Small, Zero, Negative Small, Negative Medium, Negative Big

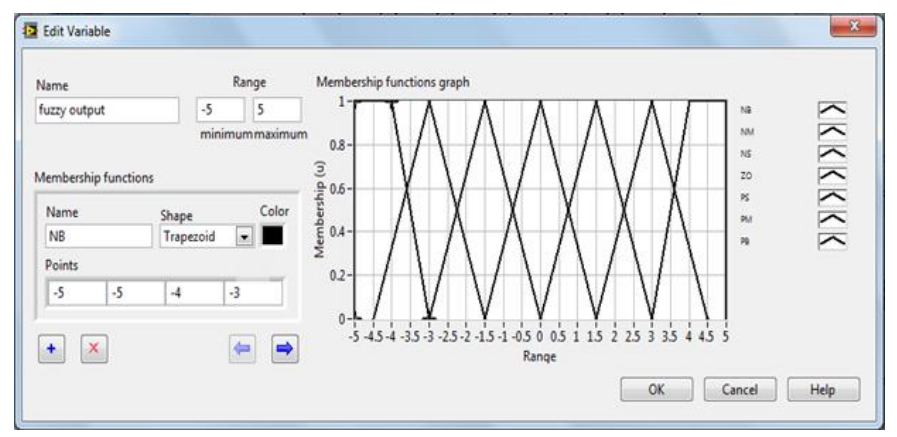

Fig8: membership function for fuzzy output

\section{The Rule Base Table}

Rules describe, in words, the relationships between input and output linguistic variables based on their linguistic terms The total number $\mathrm{N}$ of possible rules for a fuzzy system is defined by the following equation:

$$
\mathrm{N}=\mathrm{p} 1 \times \mathrm{p} 2 \times \ldots \times \mathrm{pn}
$$

where pn is the number of linguistic terms for the input linguistic variable $\mathrm{n}$. If each input linguistic variable has the same number of linguistic terms, the total number $\mathrm{N}$ of possible rules is defined by the following equation:

$$
\mathrm{N}=\mathrm{p}^{\wedge} \mathrm{m}
$$

where $\mathrm{p}$ is the number of linguistic terms for each input linguistic variable and $\mathrm{m}$ is the number of input linguistic variables.Plotting a rule base in matrix form, is helpful for detecting inconsistencies, such as contradictory rules.
However, plotting a rule base in matrix form is efficient only for small rule bases. Detecting inconsistencies in large rule bases is difficult. For fuzzy systems with numerous controller inputs, you can use cascading fuzzy systems to avoid large rule bases. The rule base table is shown below:

\begin{tabular}{|c|c|c|c|c|c|c|c|}
\hline $\begin{array}{l}\text { ERROR } \\
\text { RATE } \\
\text { ERROR }\end{array}$ & $\overline{\mathrm{PB}}$ & $\mathrm{PM}$ & PS & $\mathrm{ZO}$ & $\mathrm{NS}$ & $\mathrm{NM}$ & NB \\
\hline PB & PB & PB & PB & PB & PM & PM & PS \\
\hline PM & PB & PB & PB & PB & $\begin{array}{l}\mathrm{PB} \\
\end{array}$ & PM & PS \\
\hline PS & PB & PB & PB & PB & PM & PS & NM \\
\hline $\mathrm{ZO}$ & PB & $\mathrm{PM}$ & $\mathrm{PM}$ & $\mathrm{PM}$ & PS & $\mathrm{ZO}$ & NS \\
\hline NS & PM & PS & $\mathrm{ZO}$ & NS & NM & NB & NB \\
\hline $\mathrm{NM}$ & $\mathrm{PM}$ & $\mathrm{ZO}$ & NS & NM & NB & NB & NB \\
\hline NB & $\overline{\mathrm{ZO}}$ & NS & NM & NB & NB & NB & NB \\
\hline
\end{tabular}

Table 2: rule base table

\section{DEVELOPING FUZZY LOGIC CONTROLLER}

Fuzzy controllers are used to control fuzzy systems. Most traditional control algorithms require a mathematical model of the system you want to control. However, many physical systems are difficult or impossible to model mathematically. In addition, many processes are either nonlinear or too complex for you to control with traditional strategies. However, if you can describe a control strategy qualitatively, you can use fuzzy logic to create a fuzzy controller that emulates a heuristic ruleof-thumb strategy. After a fuzzy controller fuzzifies the input values of a fuzzy system,the fuzzy controller uses the corresponding input linguistic terms and rule base to determine the resulting linguistic terms of the output linguistic variables. The block diagram of a fuzzy controller has been shown in Fig 5.2 .

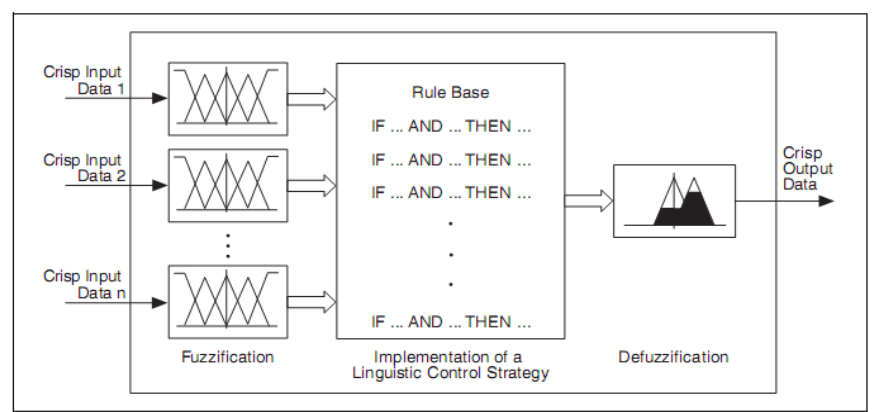

Fig9: fuzzy logic block diagram

The objective of the fuzzy controller in our project is to estimate the amount of current supplied to the heating rod and 
the inlet flow rate to achieve the desired water temperature. The basic steps in designing a fuzzy logic controller are as follows:

$$
\text { CoA }=\frac{\int_{\text {max }}^{x_{\max }} f(x) \cdot x d x}{\int_{\max }^{x_{\max }} f(x) d x}
$$

1. Identifying the input and output variables.

2.Partitioning the interval of each input and output into number of fuzzy subsets, assigning each a linguistic label.

3.Determining a membership function for each fuzzy subset.

4.Assigning the fuzzy relationship between the "input fuzzy subsets" on one hand and the "output fuzzy sets on the other hand,thus forming the Rule-Base.

5.Interpreting the rules using fuzzy "AND" and "OR" operators. In fuzzy systems, more than one rule may fire at the same time ,but with varied strengths.

6.Translating the processed fuzzy data into the crisp data suitable for real world applications.

\section{FUZZIFICATION}

Fuzzification is the process of associating crisp, or numerical, input values with the linguistic terms of the corresponding input linguistic variables. For example, a fuzzy controller might associate the temperature reading from a thermometer with the linguistic terms cold, moderate, and hot for the current temperature linguistic variable. Depending on the membership functions for the linguistic terms, the temperature value might correspond to one or more of the linguistic terms.

\section{DEFUZZIFICATION}

Defuzzification is the process of converting the degrees of membership of output linguistic variables within their linguistic terms into crisp numerical values. A fuzzy controller can use one of several mathematical methods to perform defuzzification. . The most accurate defuzzification method for a fuzzy controller varies based on the control application.

. Some of the popular methods are:

1) Max membership principle

2) Centroid method

3) Weighted average method

4) Mean-max membership

\section{The Centroid Method}

The 'Centroid method' is the most prevelant of all defuzzification methods and hence it has been used. In the
Centroid or Center of Area (CoA) defuzzification method, also called the Center of Gravity (CoG) method, the fuzzy controller first calculates the area under the scaled membership functions and within the range of the output variable. The fuzzy logic controller then uses the following equation to calculate the geometric center of this area.,

where $\mathrm{CoA}$ is the center of area, $\mathrm{x}$ is the value of the linguistic variable, and $x \min$ and $x \max$ represent the range of the linguistic variable.The Center of Area defuzzification method effectively calculates the best compromise between multiple output linguistic terms.

An important aspect of a defuzzification method is the continuity of the output signal. Consider a fuzzy system with a complete rule base and overlapping membership functions. A defuzzification method is continuous if an arbitrary small change of an input value never causes an abrupt change in the output signal. In this respect, the centroid defuzzification method is most suitable, because, assuming overlapping output membership functions, the best compromise does not jump to a different value with a small change to the inputs.

\section{TEST SYSTEM}

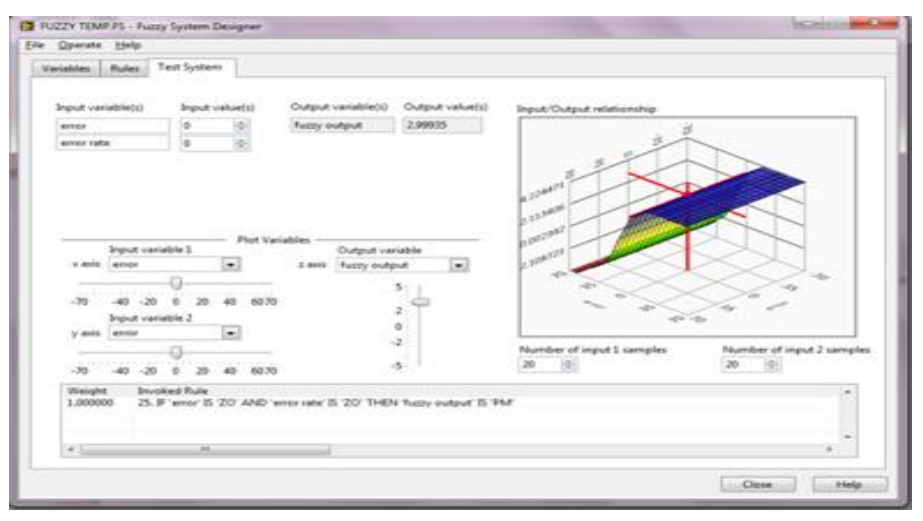

Fig 10: test system window

The final step in designing fuzzy controller is using the test system to check the accuracy of the defuzzified value. The test system of the LabVIEW is used to test the relationship between the input and output values of a fuzzy system in order to validate the rule base of the fuzzy system. In the test system the values of the input variables are entered manually. The controller then calculates the corresponding weights of the inputs. Based on the antecedent connective and consequent implication the weight of the output is found out. The test system also displays the rules invoked. The test system gives an option to select the plot variables. Based on these selections the input/output relationship is mapped. If needed the input and output variables can be altered to achieve accuracy. Finally once the output has been obtained the controller is saved. This file is loaded into the program for processing of the input and obtaining the output 


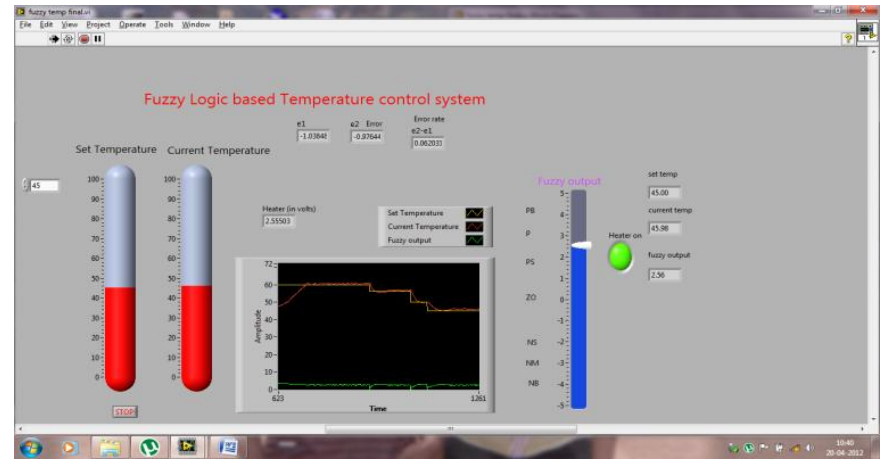

Fig 11: result window1

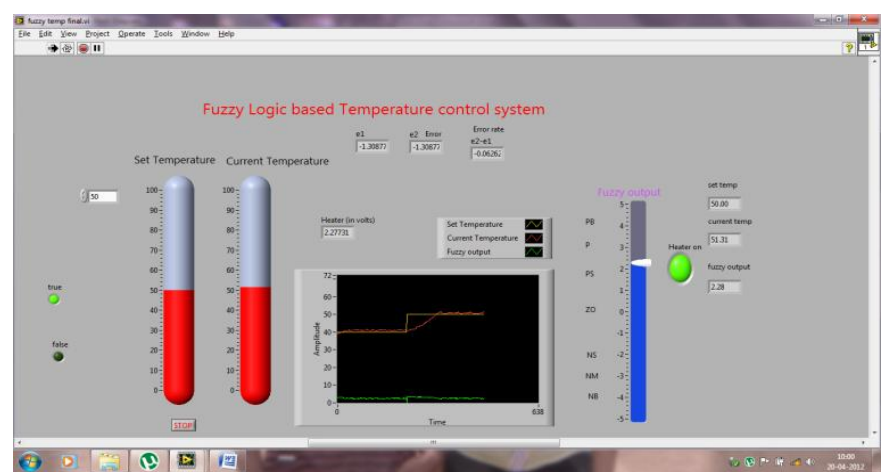

Fig 12: result window2

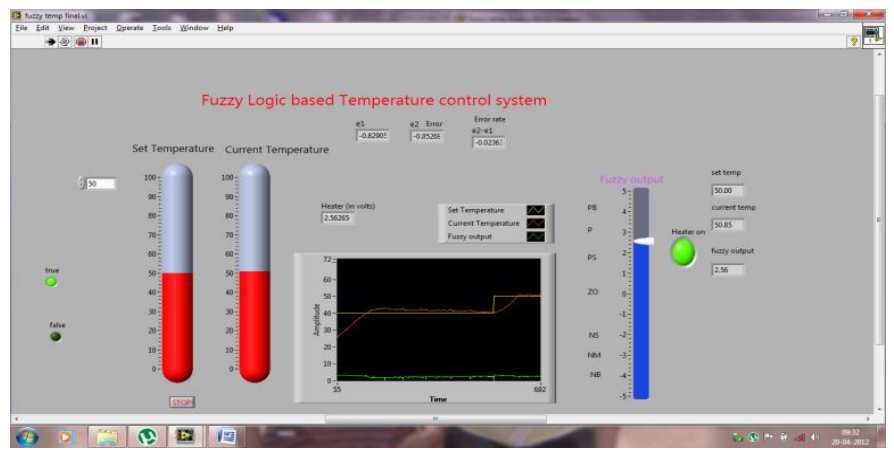

Fig 13: result window3

\section{CONCLUSIONS}

The proposed project helps in efficient monitoring and control of water temperature in the tank with the help of fuzzy controller designed in LabVIEW software. This system attempts to correct the error between the measured temperature and the desired set point thus achieving efficient temperature control. The need for today's industry is a real time monitoring and control of the various parameters in a simpler manner with easy identification and rectification of errors.As the existing system's cooling process is time consuming, speeding up the cooling process is the need of the hour which the proposed system fulfills.

\section{REFERENCES}

[1] "Chemical process control" by George Stephanopoulos, Pearson Education Publications 2009.

[2] " Design and construction of a LabVIEW based Temperature controller with using Fuzzy Logic" by A.Sertaç Sunay, Onur Koçak, Ersin Kamberli, Cengiz Koçum.

[3] "Electrical \&Electronic Measurements and Instrumentation", A.K.Sawhney, Dhanpat Rai Publication, $7^{\text {th }}$ edition 2005.

[4] "Fuzzy sets and fuzzy logic theory and applications", George J Klir

[5] "Fuzzy Logic Toolkit User Manual”, www.ni.com

[6] www.pacontrol.com/fuzzy.html

[7] www.techteach.com

[8] "Simulation and Design of Fuzzy Temperature Control for Heating and Cooling Water System", Zaid Amin Abduljabar, International Journal of Advancements in Computing Technology, Volume III, May 2011 